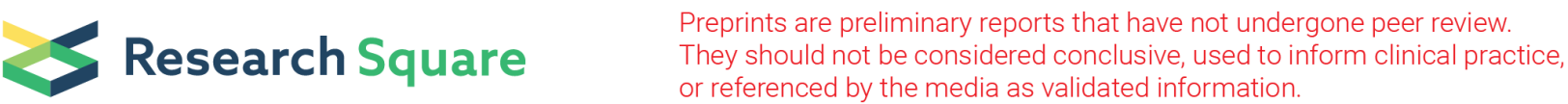

\section{Longitudinal Change of Circulating Tumor Cells During Chemoradiation Therapy and Its Correlation with Prognosis in Advanced Non-Small Cell Lung Cancer Patients}

Jun Liu

Changzhou Tumor Hospital Affiliated to Soochow University

Yongping Liu

Changzhou Tumor Hospital Affiliated to Soochow University

Cheng Gu

Changzhou Tumor Hospital Affiliated to Soochow University

Lei Zhang

Changzhou Tumor Hospital Affiliated to Soochow University

Xujing Lu ( $\nabla$ xuzhangdi56987@163.com )

Changzhou Tumor Hospital Affiliated to Soochow University https://orcid.org/0000-0003-1457-2061

\section{Research}

Keywords: circulating tumor cells, advanced non-small cell lung cancer, chemoradiation, treatment response, progression-free survival, overall survival

Posted Date: June 26th, 2020

DOI: https://doi.org/10.21203/rs.3.rs-37672/v1

License: (c) (i) This work is licensed under a Creative Commons Attribution 4.0 International License.

Read Full License 


\section{Abstract}

Background: This study aimed to investigate the association of circulating tumor cells (CTCs) change during chemoradiation with the treatment response and survival profiles in advanced non-small cell lung cancer (NSCLC) patients.

Methods: 58 advanced NSCLC patients underwent concurrent chemoradiation were enrolled, then their peripheral blood samples were collected pre-chemoradiation and at 1 months post-chemoradiation to assess the CTCs using a CTC-Biopsy system. Moreover, CTCs were classified as CTCs positive and CTCs negative according to CTCs' count, and CTCs' change was calculated. Additionally, response of chemoradiation was evaluated at 1 months post-chemoradiation, then progression-free survival (PFS) and overall survival (OS) were assessed.

Results: Pre-chemoradiation CTCs positive was associated with increased TNM stage, but not correlated with other clinicopathologic characteristics. After chemoradiation, the CTCs' number (1.0 (0.0-3.0) vs. 4.0 (2.0-10.0)) and the percentage of CTCs positive cases (37.9\% vs. $77.6 \%)$ were both decreased compared with those prior to chemoradiation. Regarding treatment response, pre-chemoradiation CTCs positive was associated with lower partial response; post-chemoradiation CTCs positive was associated with reduced disease control rate; while CTCs' change during chemoradiation was not associated with treatment response. Kaplan-Meier curves showed that post-chemoradiation CTCs positive and increased CTCs' number during chemoradiation were associated with reduced PFS, then multivariate Cox's regression analysis disclosed that they independently predicted decreased PFS. However, no correlation of CTCs status or CTCs' change with OS was observed.

Conclusions: Longitudinal monitoring of CTCs may provide important reflection for the prognosis in chemoradiation treated advanced NSCLC patients.

\section{Background}

Lung cancer, with $80-85 \%$ being non-small cell lung cancer (NSCLC), remains the main cause of cancerrelated death worldwide $[1,2]$. More than one-third of all newly diagnosed lung cancers occur in China, making it a large social and economic burden [3]. For the NSCLC patients diagnosed at early stage, surgery is commonly used to resect the tumor, while most NSCLC patients are diagnosed with advancedstage disease owing to the inadequate screening and late onset of clinical symptoms; then, for the advanced-stage disease, patients with gene mutations (such as mutation in the epidermal growth factor receptor (EGFR) gene) may benefit from targeted therapy (such as tyrosine kinase inhibitors (TKIs) of EGFR), whereas patients unavailable for target therapy have to receive chemotherapy alone or in combination with radiation therapy, by which the efficacy is still far away from satisfactory $[4,5]$.

Circulating tumor cells (CTCs) are the tumor cells that originate from either primary tumor or its metastasis circulating in the peripheral blood [6]. Detection, monitoring, and molecular investigation of CTCs offer a meaningful and noninvasive way that can help patients to customize individual treatment 
plans and have a better surveillance for tumor progression [3, 7]. As to NSCLC, a few investigations disclose the correlation of CTCs with progression or prognosis in advanced NSCLC patients [8-10]. For instance, one study shows that high baseline CTCs level is associated with poorer treatment response and shorter survival profiles in TKI or chemotherapy treated advanced NSCLC patients [8]. Also, a study displays that baseline CTCs level is correlated with decreased PFS and OS in TKI-treated advanced NSCLC patients [10]. Another study displays that higher post-treatment CTCs level correlates with worse treatment response in chemotherapy treated advanced NSCLC patients [9]. However, the indications from these previous studies are restricted to the patients treated with targeted therapy or chemotherapy alone, and they just focus on the reflection of prognosis by pre-treatment or post-treatment CTCs measurement in advanced NSCLC patients, but limited evidence uncovers the role of CTCs' change during chemoradiation in advanced NSCLC patients. To address this problem, we paid attention to the longitude change of CTCs in chemoradiation-treated advanced NSCLC patients, and aimed to investigate the association of CTCs' change during chemoradiation with the treatment response and survival profiles in advanced NSCLC patients.

\section{Materials And Methods}

\subsection{Patients}

From July 2016 to June 2019, a total of 58 advanced NSCLC patients who underwent concurrent chemoradiation in Changzhou Tumor Hospital Affiliated to Soochow University were consecutively recruited in this study. The inclusion criteria were: (i) diagnosed as NSCLC; (ii) aged above 18 years old; (iii) TNM stage IIIB-IV; (iv) Eastern Cooperative Oncology Group (ECOG) score 0-2; (v) unable to receive resection which was evaluated by the Thoracic Malignancy Multi-disciplinary Clinic; (vi) schedule to receive concurrent chemoradiation. Patients had history of other malignancies, had severe infection, severe liver/kidney dysfunction or severe cardiovascular disease were excluded. This study was approved by the Institutional Review Board of Changzhou Tumor Hospital Affiliated to Soochow University. All patients or their family members provided written informed consents.

\subsection{Data collection}

After enrollment, patients' clinical characteristics were recorded, which included age, gender, smoke status, ECOG score, histological type, TNM stage, carcinoembryonic antigen (CEA) level, carbohydrate antigen 125 (CA125) level, cytokeratin 19 (CYFRA21-1) level and neuron-specific enolase (NSE) level.

\subsection{Treatment}

All patients received concurrent chemoradiation. The radiotherapy was delivered to the primary tumor and nodal metastatic sites or oligo-metastatic site with the basis of size criteria as follows: 5250-6000 cGy in 15-20 fractions of external beam Intensity Modulated Radiation Therapy (IMRT) (300-350 cGy per fraction, 5 fraction per week) with Varian Clinac IX Linear Accelerator (Varian Medical Systems, American). The chemotherapy was carried out as one of the follows regimens according to NCCN clinical 
practice guidelines in Oncology: Non-Small Cell Lung Cancer (Version 4. 2016): (i) cisplatin $50 \mathrm{mg} / \mathrm{m}^{2}$ on days $1,8,29$, and 36 ; etoposide $50 \mathrm{mg} / \mathrm{m}^{2}$ days 1-5, 29-33; (ii) paclitaxel $45-50 \mathrm{mg} / \mathrm{m}^{2}$ weekly and carboplatin area under the curve (AUC 2).

\subsection{CTCs assessment}

Peripheral blood samples $(5 \mathrm{~mL})$ of patients were collected into Ethylene Diamine Tetraacetic Acid (EDTA) tubes at 24 hours pre-chemoradiation and 1 months post-chemoradiation, respectively. The collected blood samples were processed immediately to assess the CTCs using a CTC-Biopsy system (Wuhan YZY Medical Science \& Technology Co., Ltd., China). CTCs were gathered based on isolation by size of epithelial tumor cells (ISET) technology and separated with the use of Romanowsky staining, which were performed according to a previous report [11]. Then, the stained cells were observed at $100 \mathrm{X} / 200 \mathrm{X}$ original magnification for cell counting, further observed at $630 \mathrm{X} / 1000 \mathrm{X}$ original magnification with oil immersion for detailed cytomorphologic analysis [11]. The CTCs were identified by the presence of at least 4 of the following criteria: (i) irregular nuclear borders; (ii) nuclear-cytoplasmic ratio>0.8; (iii) nuclear larger than $18 \mu \mathrm{m}$; (iv) hyperchromatic and uneven nucleus; (v) thickened and wrinkled nuclear membrane; (vi) nuclear deviation, or large nucleoli, or abnormal nuclear division. CTCs positive was defined as the count of CTCs in $5 \mathrm{~mL}$ peripheral blood sample $\geq 2$ [12]. CTCs' change was calculated by subtracting CTCs post-chemoradiation from CTCs pre-chemoradiation, and the negative value was defined as CTCs up, the positive and unchanged value was defined as CTCs down/unchanged.

\subsection{Follow-up and outcomes assessment}

All patients received serial radiographic surveillance with either positron emission tomography-computed tomography (PET/CT) or intravenous contrast CT scans at 1 months post-chemoradiation, every 3 months in the first 2 years, then every 4-6 months. The response of chemoradiation were evaluated at 1 months post-chemoradiation according to the response evaluation criteria in solid tumors (Version 1.1) [13], which included: (i) complete response (CR), disappearance of all target lesions; (ii) partial response (PR), at least a $30 \%$ decrease in the sum of diameters of target lesions, taking as reference the baseline sum diameters; (iii) progressive disease (PD), at least a $20 \%$ increase in the sum of diameters of target lesions, taking as reference the smallest sum on study; (iv) stable disease (SD), neither sufficient shrinkage to qualify for PR nor sufficient increase to qualify for PD. Meanwhile, objective response rate (ORR) was defined as the percentage of patients with CR or PR, and disease control rate (DCR) was defined as the percentage of patients with CR, PR or SD. The disease status and survival status of patients were recorded regularly by visit or telephone. Progression-free survival (PFS) was calculated from the date of initiation of chemoradiation to the date of progression or death, and for the patients not known to have progression or died at last follow-up, they were censored on the date of last examined. Overall survival (OS) was calculated from the date of initiation of chemoradiation to the date of death, and for the patients not known to have died at last follow-up, they were censored on the date of last known to be alive. 


\subsection{Statistical analysis}

Statistical analyses were performed using SPSS 22.0 software (IBM, USA). Figures were plotted using GraphPad Prism 7.01 software (GraphPad Software, USA). Comparison between pre-chemoradiation CTCs and post-chemoradiation CTCs was determined by Wilcoxon signed-rank test or McNemar's test. Comparison between CTCs negative patients and CTCs positive patients was determined by Chi-square test. Comparison between CTCs down/unchanged patients and CTCs up patients was determined by Chisquare test or Fisher's exact test. PFS and OS were illustrated using Kaplan-Meier curves, and the differences of PFS and OS between CTCs negative patients and CTCs positive patients, or between CTCs down/unchanged patients and CTCs up patients, were determined by Log-rank test. Factors independently predicting PFS and OS were analyzed by backward stepwise multivariate Cox's proportional hazard regression model. $P$ value $<0.05$ was considered significant.

\section{Results}

\subsection{Characteristics of NSCLC patients}

58 NSCLC patients (including 10 (17.2\%) females and 48 (82.8\%) males) with mean age of $63.0 \pm$ 12.0 years were enrolled (Table 1). There were 10 (17.2\%), $36(62.1 \%)$ and $12(20.7 \%)$ patients showed ECOG score of 0,1 and 2 respectively. Regarding the histological type, 35 (60.3\%) patients were identified as SCC and 23 (39.7\%) patients were identified as ADC. As to TNM stage, 30 (51.7\%) patients were with TNM stage $\otimes B$ and 28 (48.3\%) patients were with TNM stage $\otimes$. Besides, other detailed information of tumor biomarkers such as CEA, CA125, CYFRA21-1 and NSE was exhibited in Table 1. 
Table 1

Patients' characteristics

\section{Items}

Age, mean \pm SD

$<65$ years, No. $(\%)$

$\geq 65$ years, №. (\%)

Gender, No. (\%)

Male

Female

Smoking, No. (\%)

ECOG score, No. (\%)

0

1

2

Histological type, No. (\%)

SCC

$A D C$

TNM stage, No. (\%)

IIIB

IV

CEA, No. (\%)

Normal $(<5 \mathrm{ng} / \mathrm{mL})$

Abnormal ( $\geq 5 \mathrm{ng} / \mathrm{mL}$ )

CA125, №. (\%)

Normal $(<35 \mathrm{U} / \mathrm{mL})$

Abnormal $(\geq 35 \mathrm{U} / \mathrm{mL})$

CYFRA21-1, No. (\%)

Normal $(<3.3 \mathrm{ng} / \mathrm{mL})$

NSCLC, non-small cell lung cancer; SD, standard deviation; ECOG, Eastern Cooperative Oncology Group; SCC, squamous cell carcinoma; ADC, adenocarcinoma; CEA, carcinoembryonic antigen; CA125, carbohydrate antigen 125; CYFRA21-1, cytokeratin 19; NSE, neuron-specific enolase.

\section{NSCLC patients $(\mathrm{N}=58)$}

$63.0 \pm 12.0$

$30(51.7)$

$28(48.3)$

$48(82.8)$

$10(17.2)$

$35(60.3)$

10 (17.2)

36 (62.1)

12 (20.7)

35 (60.3)

23 (39.7)

30 (51.7)

28 (48.3)

49 (84.5)

9 (15.5)

51 (87.9)

7 (12.1)

$33(56.9)$ 


\begin{tabular}{|lc|}
\hline Items & NSCLC patients $(\mathbf{N}=\mathbf{5 8})$ \\
\hline Abnormal $(\geq 3.3 \mathrm{ng} / \mathrm{mL})$ & $25(43.1)$ \\
\hline NSE, No. $(\%)$ & $45(77.6)$ \\
\hline Normal $(<17 \mathrm{ng} / \mathrm{mL})$ & $13(22.4)$ \\
\hline Abnormal $(\geq 17 \mathrm{ng} / \mathrm{mL})$ & $\begin{array}{l}\text { NSCLC, non-small cell lung cancer; SD, standard deviation; ECOG, Eastern Cooperative Oncology } \\
\text { Group; SCC, squamous cell carcinoma; ADC, adenocarcinoma; CEA, carcinoembryonic antigen; CA125, } \\
\text { carbohydrate antigen 125; CYFRA21-1, cytokeratin 19; NSE, neuron-specific enolase. }\end{array}$ \\
\hline
\end{tabular}

\subsection{Association of pre-chemoradiation CTCs with clinicopathologic characteristics}

According to the pre-chemoradiation CTCs level, 45 patients were categorized as CTCs positive patients and 13 patients were categorized as CTCs negative patients (Table 2). CTCs positive was associated with increased TNM stage in NSCLC patients $(P=0.007)$. Whereas no correlation of CTCs status with age $(P=$ $0.421)$, gender $(P=0.301)$, smoking $(P=0.457)$, ECOG score $(P=0.189)$, histological type $(P=0.457)$, CEA $(P=0.988)$, CA125 $(P=0.167)$, CYFRA21-1 $(P=0.701)$ or NSE $(P=0.490)$ was observed in NSCLC patients. 
Table 2

Correlation of pre-chemoradiation CTCs with clinicopathologic characteristics

\begin{tabular}{|c|c|c|c|}
\hline \multirow[t]{2}{*}{ Items } & \multicolumn{2}{|c|}{ Pre-chemoradiation CTCs } & \multirow[t]{2}{*}{$P$ value } \\
\hline & Positive $(n=45)$ & Negative $(n=13)$ & \\
\hline Age, No. (\%) & & & 0.421 \\
\hline$<65$ years & $22(48.9)$ & $8(61.5)$ & \\
\hline$\geq 65$ years & $23(51.1)$ & $5(38.5)$ & \\
\hline Gender, No. (\%) & & & 0.301 \\
\hline Male & $36(80.0)$ & $12(92.3)$ & \\
\hline Female & $9(20.0)$ & $1(7.7)$ & \\
\hline Smoking, No. (\%) & & & 0.457 \\
\hline No & $19(42.2)$ & $4(30.8)$ & \\
\hline Yes & $26(57.8)$ & $9(69.2)$ & \\
\hline ECOG score, No. (\%) & & & 0.189 \\
\hline $0 / 1$ & $34(75.6)$ & $12(92.3)$ & \\
\hline 2 & $11(24.4)$ & $1(7.7)$ & \\
\hline Histological type, No. (\%) & & & 0.457 \\
\hline SCC & $19(42.2)$ & $4(30.8)$ & \\
\hline$A D C$ & $26(57.8)$ & $9(69.2)$ & \\
\hline TNM stage, No. (\%) & & & 0.007 \\
\hline IIIB & $19(42.2)$ & $11(84.6)$ & \\
\hline IV & $26(57.8)$ & $2(15.4)$ & \\
\hline CEA, No. (\%) & & & 0.988 \\
\hline Normal (<5 ng/mL) & $38(84.4)$ & $11(84.6)$ & \\
\hline Abnormal ( $\geq 5 \mathrm{ng} / \mathrm{mL})$ & $7(15.6)$ & $2(15.4)$ & \\
\hline CA125, №. (\%) & & & 0.167 \\
\hline Normal (<35 U/mL) & $41(91.1)$ & $10(76.9)$ & \\
\hline
\end{tabular}

Comparison was determined by Chi-square test. CTCs, circulating tumor cells; ECOG, Eastern Cooperative Oncology Group; SCC, squamous cell carcinoma; ADC, adenocarcinoma; CEA, carcinoembryonic antigen; CA125, carbohydrate antigen 125; CYFRA21-1, cytokeratin 19; NSE, neuronspecific enolase. 


\begin{tabular}{|c|c|c|c|}
\hline \multirow[t]{2}{*}{ Items } & \multicolumn{2}{|c|}{ Prechemoradiation CTCs } & \multirow[t]{2}{*}{$P$ value } \\
\hline & Positive $(n=45)$ & Negative $(n=13)$ & \\
\hline Abnormal ( $\geq 35 \mathrm{U} / \mathrm{mL})$ & $4(8.9)$ & $3(23.1)$ & \\
\hline \multicolumn{3}{|l|}{ CYFRA21-1, No. (\%) } & 0.701 \\
\hline Normal (<3.3 ng/mL) & $25(55.6)$ & $8(61.5)$ & \\
\hline Abnormal ( $\geq 3.3 \mathrm{ng} / \mathrm{mL}$ ) & $20(44.4)$ & $5(38.5)$ & \\
\hline \multicolumn{3}{|l|}{ NSE, No. (\%) } & 0.490 \\
\hline Normal (<17 ng/mL) & $34(75.6)$ & $11(84.6)$ & \\
\hline Abnormal ( $\geq 17 \mathrm{ng} / \mathrm{mL}$ ) & $11(24.4)$ & $2(15.4)$ & \\
\hline \multicolumn{4}{|c|}{$\begin{array}{l}\text { Comparison was determined by Chi-square test. CTCs, circulating tumor cells; ECOG, Eastern } \\
\text { Cooperative Oncology Group; SCC, squamous cell carcinoma; ADC, adenocarcinoma; CEA, } \\
\text { carcinoembryonic antigen; CA125, carbohydrate antigen 125; CYFRA21-1, cytokeratin 19; NSE, neuron- } \\
\text { specific enolase. }\end{array}$} \\
\hline
\end{tabular}

\subsection{Comparison between post-chemoradiation CTCs and pre-chemoradiation CTCs}

The number of post-chemoradiation CTCs was decreased than the number of pre-chemoradiation CTCs $(1.0(0.0-3.0)$ vs. $4.0(2.0-10.0), P=0.004)$ (Table 3$)$, meanwhile, the percentage of post-chemoradiation CTCs positive cases was reduced compared with percentage of pre-chemoradiation CTCs positive cases $(P<0.001)$.

Table 3

Comparison between pre-chemoradiation CTCs and post-chemoradiation CTCs

\begin{tabular}{|l|l|l|l|}
\hline Items & Pre-chemoradiation & Post-chemoradiation & $P$ value \\
\hline Number of CTCs, median (IQR) & $4.0(2.0-10.0)$ & $1.0(0.0-3.0)$ & 0.004 \\
\hline CTCs status, No. (\%) & & & $<0.001$ \\
\hline Positive & $45(77.6)$ & $22(37.9)$ & \\
\hline Negative & $13(22.4)$ & $36(62.1)$ & \\
\hline $\begin{array}{l}\text { Comparison was determined by Wilcoxon signed-rank test or McNemar's test. CTCs, circulating tumor } \\
\text { cells; IQR, interquartile range. }\end{array}$ & \\
\hline
\end{tabular}

\subsection{Association of CTCs status with treatment response to chemoradiation}

Pre-chemoradiation CTCs positive was associated with lower PR $(P=0.039)$, while no correlation of prechemoradiation CTCs status with CR $(P=0.439), \mathrm{SD}(P=0.326), \mathrm{PD}(P=0.320), \mathrm{ORR}(P=0.073)$ or DCR $(P=0.320)$ was observed in NSCLC patients (Table 4). Moreover, post-chemoradiation CTCs positive was associated with increased PD $(P=0.025)$ and reduced $\mathrm{DCR}(P=0.025)$, while no correlation of postchemoradiation CTCs status with CR $(P=1.000)$, PR $(P=0.837), \mathrm{SD}(P=0.141)$ or ORR $(P=0.940)$ was observed in NSCLC patients. Additionally, the CTCs' change during chemoradiation was not associated with treatment response in advanced NSCLC patients (all $P>0.05$ ). 
Table 4

Correlation of CTCs status with treatment response

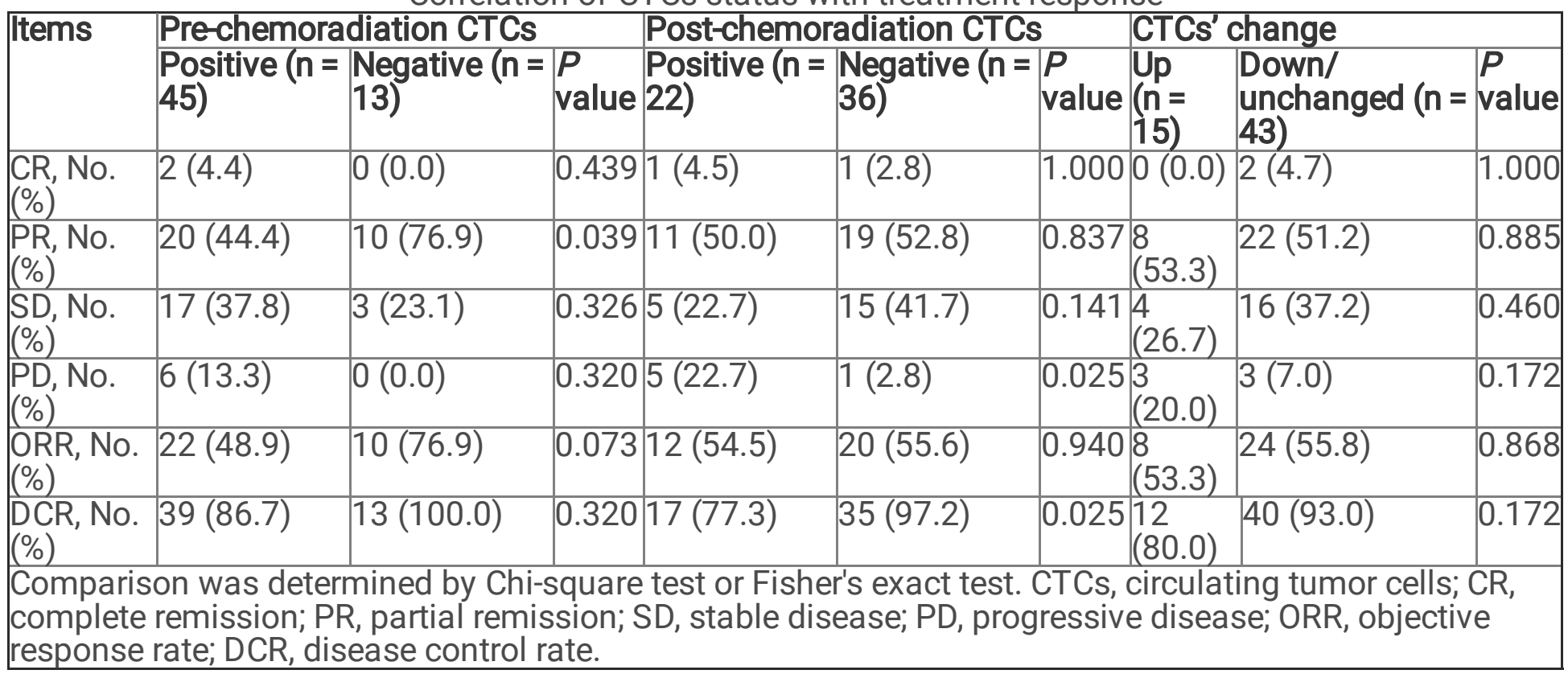

\subsection{Association of CTCs status with PFS}

The pre-chemoradiation CTCs positive showed a trend to be correlated with shorter PFS, while no significant difference was observed $(P=0.053)($ Fig. 1A). Moreover, post-chemoradiation CTCs positive $(P=0.016)$ (Fig. 1B) and CTCs up after treatment $(P=0.034)$ (Fig. 1C) were associated with reduced PFS in NSCLC patients.

\subsection{Association of CTCs status with OS}

No correlation of pre-chemoradiation CTCs status $(P=0.584)$ (Fig. 2A), post-chemoradiation CTCs status $(P=0.131)$ (Fig. 2B) or CTCs' change with OS $(P=0.292)$ (Fig. 2C) was observed in NSCLC patients.

\subsection{Analysis of factors affecting PFS and OS}

Backward stepwise multivariate Cox's proportional hazard regression model analysis displayed that prechemoradiation CTCs positive ( $P=0.008)$ (HR: $3.346,95 \% \mathrm{Cl}: 1.379-8.121)$ was an independent predictive factor for decreased PFS, so did CTCs' change (up vs. down/unchanged) $(P=0.002)(\mathrm{HR}$ : 3.130, 95\% Cl: 1.504-6.516) and NSE (abnormal vs. normal) ( $P=0.026)$ (HR: $2.199,95 \%$ Cl: $1.099-4.400)$ (Table 5). In addition, NSE (abnormal vs. normal) ( $P=0.022)$ (HR: $2.504,95 \% \mathrm{Cl}: 1.144-5.483)$ was an independent factor predicting worse OS in NSCLC patients. 
Table 5

Independent factors predicting PFS and OS

\begin{tabular}{|c|c|c|c|c|}
\hline \multirow[t]{2}{*}{ thems } & \multicolumn{4}{|c|}{$\begin{array}{l}\text { Backward stepwise multivariate Cox's proportional hazard } \\
\text { regression model }\end{array}$} \\
\hline & $P$ value & HR & \multicolumn{2}{|l|}{$95 \% \mathrm{Cl}$} \\
\hline Lower & & Higher & & \\
\hline \multicolumn{5}{|l|}{ PFS } \\
\hline Pre-chemoradiation CTCs positive & 0.008 & 3.346 & 1.379 & 8.121 \\
\hline $\begin{array}{l}\text { CTCs' change (up vs. } \\
\text { down/unchanged) }\end{array}$ & 0.002 & 3.130 & 1.504 & 6.516 \\
\hline NSE (abnormal vs. normal) & 0.026 & 2.199 & 1.099 & 4.400 \\
\hline \multicolumn{5}{|l|}{ OS } \\
\hline NSE (abnormal vs. normal) & 0.022 & 2.504 & 1.144 & 5.483 \\
\hline $\begin{array}{l}\text { Factors independently predicting P } \\
\text { proportional hazard regression mo } \\
\text { ratio; Cl, confidence interval; CTCs, }\end{array}$ & & yzed by & stepwi & $\begin{array}{l}\text { variate Cox's } \\
\text { HR, hazard }\end{array}$ \\
\hline
\end{tabular}

\section{Discussion}

Knowledge of metastasis may help understand the biology of CTCs in NSCLC [7, 14, 15]. Metastasis is an extremely complex and multistep process, in which the tumor cells must undergo a cellular process termed as epithelial mesenchymal transition (EMT) [7]. EMT enables the tumor cells to be motile, and also allows cells to penetrate into the lymph vessels and to circulate as a single or clusters of CTCs $[7,14$, 15]. Then, CTCs spread to distant organs and facilitate the formation of secondary sites of the tumor disease, moreover, CTCs may form disseminating tumor cells known as a settlement of CTCs in other organs, which may stay in a quiescence state or lead to an observable metastasis [16]. Thus, CTCs are regarded as potential metastasis-inducing cells. In addition, previous data demonstrate that the CTCs clusters have high expressions of adhesion markers (such as MPDZ and TNC genes), which endow the clusters an advantage of avoiding anoikis in the circulation, furthermore, various genetic alterations (such as epidermal growth factor receptor (EGFR), MET and B-Raf proto-oncogene (BRAF)) that correlate with resistance of chemotherapy are observed to be highly expressed on CTCs [17-19]. With these properties, monitoring CTCs may bring important indications for the progression of NSCLC.

In clinical practices, previous investigations have disclosed that CTCs provide important reflection for the clinicopathological features of lung cancer patients: one study shows the positive association of prechemoradiation CTCs' count with lymph node metastasis, distant metastasis and TNM stage in small cell lung cancer patients [20]; another study discloses that pre-chemotherapy CTCs positive is associated with lymph node metastases and larger primary tumors in early stage NSCLC patients [21]. However, the related evidence in advanced NSCLC patients is limited. In our study, we observed that the prechemoradiation CTCs positive was associated with increased TNM stage in advanced NSCLC patients, which might due to: higher CTCs' count indicated more cancer cells could be displaced to distant site, which was a crucial step promoting the process of metastasis and leading to elevated TNM stage, thus pre-chemoradiation CTCs positive status was positively correlated with TNM stage in advanced NSCLC patients $[7,14]$. 
Regarding the detection of CTCs' change during treatment in advanced NSCLC patients, it is reported in only two studies: one study displays that there is no change of CTCs' number among the time points of post-first cycle of chemotherapy, post-second cycle of chemotherapy and post-third cycle of chemotherapy in advanced NSCLC patients [9]; another study shows that CTCs' number is increased in majority of TKI-treated advanced NSCLC patients [10]. However, related investigation in chemoradiationtreated advanced NSCLC patients is seldomly reported. Our study compared the post-chemoradiation CTCs' number and pre-chemoradiation CTCs' number, then we found that the CTCs' number and percentage of CTCs positive cases both decreased after chemoradiation compared with those prior to chemoradiation. These results might be explained by: chemotherapy in combination with radiation directly promoted apoptosis of CTCs, meanwhile, chemoradiation induced tumor necrosis, inhibited tumor progression and further attenuated the production of CTCs, thus CTCs' number and percentage CTCs positive cases were reduced after chemoradiation therapy in advanced NSCLC patients.

Moreover, assessing CTCs is a useful way for the surveillance in cancer patients' prognosis after treatment [22-25]. For example, negative association of CTCs level and treatment response has been investigated in several cancers, such as nasopharyngeal carcinoma, colorectal cancers and breast cancer [23-25]. For lung cancer, one study shows that the pre-chemoradiation CTCs is negatively associated with PR in advanced NSCLC patients [22]. Furthermore, a study displays that elevated pre-radiation CTCs and persistence of CTCs post-radiation are associated with higher recurrence risk outside the targeted treatment site in early stage NSCLC patients [26]. Another study displays that higher pre-chemoradiation CTCs level is an independent predictive factor for worse OS in small cell lung cancer patients [20]. All these studies just evaluate the correlation of pre-treatment or post-treatment CTCs level with prognosis of lung cancer patients, resulting in a paucity of evidence about the role of CTCs' change during treatment in advanced NSCLC patients, meanwhile, related evidence in chemoradiation treated advanced NSCLC patients is limited. Therefore, our study explored the association of pre-treatment CTCs, post-treatment CTCs and CTCs' change during treatment with the treatment response and survival profiles in chemoradiation treated advanced NSCLC patients. We observed that pre-chemoradiation CTCs positive was associated with decreased PR, meanwhile, post-chemoradiation CTCs positive was associated with elevated PD and reduced DCE in advanced NSCLC patients. In addition, pre-chemoradiation CTCs positive, post-chemoradiation CTCs positive and CTCs' up after treatment were associated with shorter PFS in advanced NSCLC patients. The possible reasons for these results might be as follows: (1) CTCs is be able to access the distant organs and pre-chemoradiation CTCs positive may indicate that CTCs have undergone the EMT process and tend to colonized at distant organs, facilitating the distant metastasis and indicating less possibility to achieve survive from the disease, thus pre-chemoradiation CTCs positive was associated with decreased PR and shorter PFS in advanced NSCLC patients underwent chemoradiation therapy [27-29]; (2) post-chemoradiation CTCs positive and CTCs up after treatment indicate that considerable CTCs survive from the chemoradiation therapy and may further facilitate the formation of tumors at secondary sites, leading to aggravated progression, thus post-chemoradiation CTCs positive correlated with increased PD, reduced DSE and shorter PFS in advanced NSCLC patients [17-19]. 
There were still some limitations in our study, which needed to be improved in future. Firstly, the sample size of 58 was small, which might cause a relatively low statistical efficacy, thus larger number of patients was desirable for a further study. Secondly, this was a single-center study, which might have some selection bias, and further validations of our findings in a multi-center study was needed. Thirdly, the post-chemoradiation CTCs were only detected at 1 month after treatment, whereas it would be difficult to constantly count CTCs during the follow-up.

\section{Conclusions}

In conclusion, longitudinal monitoring of CTCs may provide important reflection for the prognosis in chemoradiation treated advanced NSCLC patients.

\section{Abbreviations}

NSCLC: non-small cell lung cancer, EGFR: epidermal growth factor receptor, TKIs: tyrosine kinase inhibitors, CTCs: Circulating tumor cells, ECOG: Eastern Cooperative Oncology Group, CA125: carbohydrate antigen 125, NSE: neuron-specific enolase, IMRT: Intensity Modulated Radiation Therapy, EDTA: Ethylene Diamine Tetraacetic Acid, PET/CT: positron emission tomography-computed tomography, CR: complete response, PR: partial response, PD: progressive disease, SD: stable disease, ORR: objective response rate, DCR: disease control rate, PFS: Progression-free survival, OS: Overall survival

\section{Declarations}

\section{Ethics approval and consent to participate}

This study was approved by the Institutional Review Board of Changzhou Tumor Hospital Affiliated to Soochow University. All patients or their family members provided written informed consents.

\section{Consent for publication}

Not Applicable.

\section{Availability of data and material}

The datasets used and/or analyzed during the current study are available from the corresponding author on reasonable request.

\section{Competing interests}

The authors of this work have nothing to disclose.

\section{Funding}

Not Applicable. 
Authors' contributions

Conception and design: Xujing Lu; Collection and assembly of data: Jun Liu and Yongping Liu; Data analysis and interpretation: Cheng Gu and Lei Zhang; Manuscript writing: All authors.

Acknowledgements

None.

\section{References}

1. Bray F, Ferlay J, Soerjomataram I, Siegel RL, Torre LA, Jemal A: Global cancer statistics 2018: GLOBOCAN estimates of incidence and mortality worldwide for 36 cancers in 185 countries.CA Cancer J Clin 2018, 68:394-424.

2. Torre LA, Bray F, Siegel RL, Ferlay J, Lortet-Tieulent J, Jemal A: Global cancer statistics, 2012.CA Cancer J Clin 2015, 65:87-108.

3. Zhang Y, Zheng H, Zhan Y, Long M, Liu S, Lu J, Zang H, Fan S: Detection and application of circulating tumor cell and circulating tumor DNA in the non-small cell lung cancer.Am $J$ Cancer Res 2018, 8:2377-2386.

4. Ai X, Guo X, Wang J, Stancu AL, Joslin PMN, Zhang D, Zhu S: Targeted therapies for advanced nonsmall cell lung cancer.Oncotarget 2018, 9:37589-37607.

5. Yoneda K, Imanishi N, Ichiki Y, Tanaka F: Treatment of Non-small Cell Lung Cancer with EGFRmutations.J UOEH 2019, 41:153-163.

6. Alix-Panabieres C, Pantel K: Circulating tumor cells: liquid biopsy of cancer.Clin Chem 2013, 59:110118.

7. Kapeleris J, Kulasinghe A, Warkiani ME, Vela I, Kenny L, O'Byrne K, Punyadeera C: The Prognostic Role of Circulating Tumor Cells (CTCs) in Lung Cancer.Front Oncol 2018, 8:311.

8. Tamminga M, de Wit S, Schuuring E, Timens W, Terstappen L, Hiltermann TJN, Groen HJM: Circulating tumor cells in lung cancer are prognostic and predictive for worse tumor response in both targeted- and chemotherapy. Trans/ Lung Cancer Res 2019, 8:854-861.

9. Tong B, Xu Y, Zhao J, Chen M, Xing J, Zhong W, Wang M: Prognostic significance of circulating tumor cells in non-small cell lung cancer patients undergoing chemotherapy.Oncotarget 2017, 8:8661586624.

10. He W, Li W, Jiang B, Chang L, Jin C, Tu C, Li Y: Correlation between epidermal growth factor receptor tyrosine kinase inhibitor efficacy and circulating tumor cell levels in patients with advanced nonsmall cell lung cancer.Onco Targets Ther 2016, 9:7515-7520.

11. Vona G, Sabile A, Louha M, Sitruk V, Romana S, Schutze K, Capron F, Franco D, Pazzagli M, Vekemans M, et al: Isolation by size of epithelial tumor cells : a new method for the 
immunomorphological and molecular characterization of circulatingtumor cells. Am J Patho/ 2000, 156:57-63.

12. Krebs MG, Sloane R, Priest L, Lancashire L, Hou JM, Greystoke A, Ward TH, Ferraldeschi R, Hughes A, Clack G, et al: Evaluation and prognostic significance of circulating tumor cells in patients with nonsmall-cell lung cancer.J Clin Oncol 2011, 29:1556-1563.

13. Watanabe H, Okada M, Kaji Y, Satouchi M, Sato Y, Yamabe $Y$, Onaya H, Endo M, Sone M, Arai Y: [New response evaluation criteria in solid tumours-revised RECIST guideline (version 1.1)].Gan To Kagaku Ryoho 2009, 36:2495-2501.

14. Thiery JP: Epithelial-mesenchymal transitions in tumour progression.Nat Rev Cancer 2002, 2:442454.

15. Nurwidya F, Zaini J, Putra AC, Andarini S, Hudoyo A, Syahruddin E, Yunus F: Circulating Tumor Cell and Cell-free Circulating Tumor DNA in Lung Cancer.Chonnam Med J 2016, 52:151-158.

16. Joosse SA, Gorges TM, Pantel K: Biology, detection, and clinical implications of circulating tumor cells.EMBO Mol Med 2015, 7:1-11.

17. Rolfo C, Giovannetti E, Hong DS, Bivona T, Raez LE, Bronte G, Buffoni L, Reguart N, Santos ES, Germonpre P, et al: Novel therapeutic strategies for patients with NSCLC that do not respond to treatment with EGFR inhibitors.Cancer Treat Rev 2014, 40:990-1004.

18. Finocchiaro G, Toschi L, Gianoncelli L, Baretti M, Santoro A: Prognostic and predictive value of MET deregulation in non-small cell lung cancer.Ann Trans/ Med 2015, 3:83.

19. Marchetti A, Felicioni L, Malatesta S, Grazia Sciarrotta M, Guetti L, Chella A, Viola P, Pullara C, Mucilli F, Buttitta F: Clinical features and outcome of patients with non-small-cell lung cancer harboring BRAF mutations.J Clin Oncol 2011, 29:3574-3579.

20. Wang PP, Liu SH, Chen CT, Lv L, Li D, Liu QY, Liu GL, Wu Y: Circulating tumor cells as a new predictive and prognostic factor in patients with small cell lung cancer.J Cancer 2020, 11:2113-2122.

21. Hanssen A, Wagner J, Gorges TM, Taenzer A, Uzunoglu FG, Driemel C, Stoecklein NH, Knoefel WT, Angenendt $S$, Hauch S, et al: Characterization of different CTC subpopulations in non-small cell lung cancer.Sci Rep 2016, 6:28010.

22. Tarumi S, Gotoh M, Kasai Y, Matsuura N, Okuda M, Go T, Ishikawa S, Yokomise H: Innovative method using circulating tumor cells for prediction of the effects of induction therapy on locally advanced non-small cell lung cancer.J Cardiothorac Surg 2013, 8:175.

23. Qian Y, Wu Y, Yuan Z, Niu X, He Y, Peng J, Zhou F, Wei S, Hu D, Zhou Y: The Frequency of Circulating Tumour Cells and the Correlation with the Clinical Response to Standard Chemoradiotherapy in Locally Advanced Nasopharyngeal Carcinoma: A Prospective Study.Cancer Manag Res 2019, 11:10187-10193.

24. Chereches G, Barbos O, Buiga R, Balacescu O, lancu D, Todor N, Balacescu L, Miron N, Bejinariu N, Ciuleanu TE: Biomarkers for the early detection of relapses in metastatic colorectal cancers.J BUON 2017, 22:658-666. 
25. Ma S, Ling F, Gui A, Chen S, Sun Y, Li Z: Predictive Value of Circulating Tumor Cells for Evaluating Short- and Long-Term Efficacy of Chemotherapy for Breast Cancer.Med Sci Monit 2017, 23:48084816.

26. Frick MA, Feigenberg SJ, Jean-Baptiste SR, Aguarin LA, Mendes A, Chinniah C, Swisher-McClure S, Berman A, Levin W, Cengel KA, et al: Circulating Tumor Cells Are Associated with Recurrent Disease in Patients with Early-Stage Non-Small Cell Lung Cancer Treated with Stereotactic Body Radiotherapy.Clin Cancer Res 2020, 26:2372-2380.

27. Aceto N, Bardia A, Miyamoto DT, Donaldson MC, Wittner BS, Spencer JA, Yu M, Pely A, Engstrom A, Zhu $\mathrm{H}$, et al: Circulating tumor cell clusters are oligoclonal precursors of breast cancer metastasis.Cell 2014, 158:1110-1122.

28. Hou JM, Krebs M, Ward T, Sloane R, Priest L, Hughes A, Clack G, Ranson M, Blackhall F, Dive C: Circulating tumor cells as a window on metastasis biology in lung cancer.Am J Pathol 2011, 178:989-996.

29. Yu M, Bardia A, Wittner BS, Stott SL, Smas ME, Ting DT, Isakoff SJ, Ciciliano JC, Wells MN, Shah AM, et al: Circulating breast tumor cells exhibit dynamic changes in epithelial and mesenchymal composition.Science 2013, 339:580-584.

\section{Figures}

A

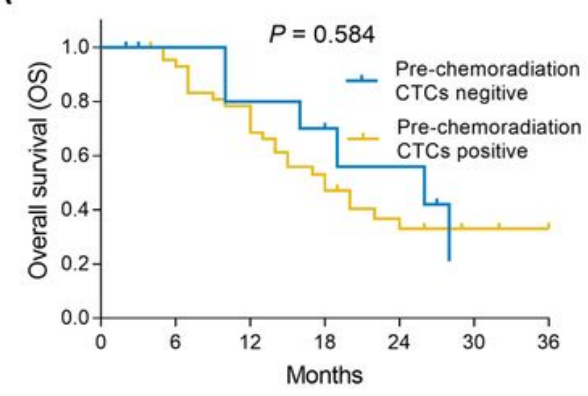

B

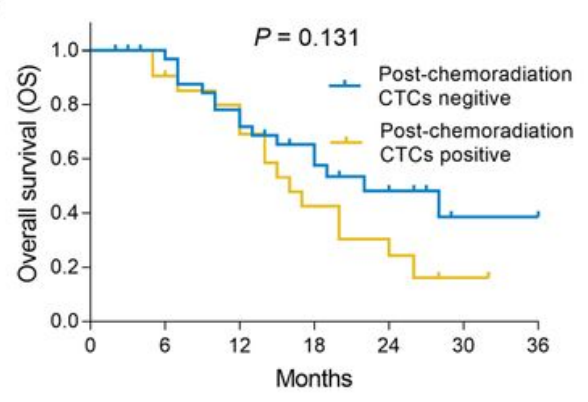

C

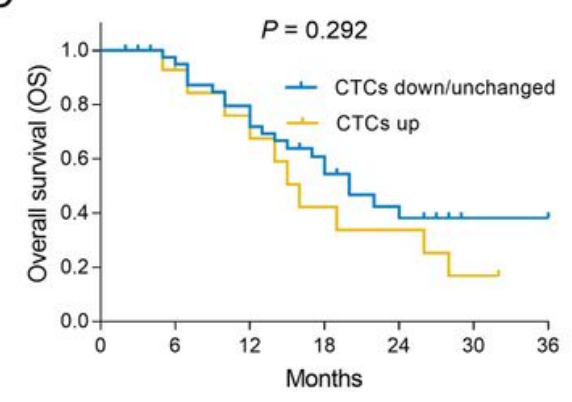

Figure 1

Correlation of CTCs with OS in NSCLC patients. Correlation of pre-chemoradiation CTCs status (A), postchemoradiation CTCs status (B) and CTCs' change (C) with OS in NSCLC patients. CTCs, circulating tumor cells; OS, overall survival; NSCLC, non-small cell lung cancer. 
A

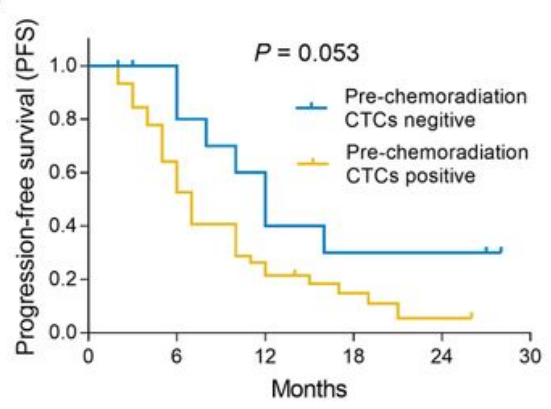

B

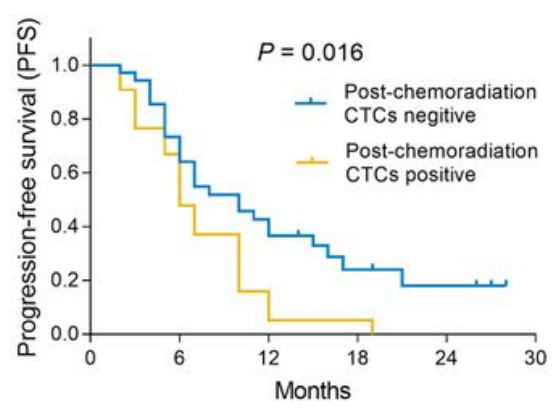

C

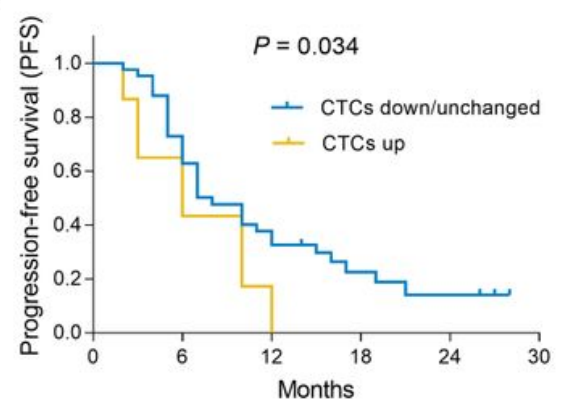

Figure 2

Correlation of CTCs with PFS in NSCLC patients. Correlation of pre-chemoradiation CTCs status (A), postchemoradiation CTCs status (B) and CTCs' change (C) with PFS in NSCLC patients. CTCs, circulating tumor cells; PFS, progression-free survival; NSCLC, non-small cell lung cancer. 